\title{
Colocalization of fast and slow timescale dynamics in the allosteric signaling protein $\mathrm{CheY}$
}

\author{
Leanna R. McDonald ${ }^{1}$, Matthew J. Whitley ${ }^{1}$, Joshua A. Boyer ${ }^{1}$, and Andrew L. Lee ${ }^{1,2}$ \\ ${ }^{1}$ Department of Biochemistry and Biophysics, School of Medicine, University of North Carolina at \\ Chapel Hill, Chapel Hill, North Carolina, 27599, USA \\ 2Division of Chemical Biology and Medicinal Chemistry, Eshelman School of Pharmacy, \\ University of North Carolina at Chapel Hill, Chapel Hill, North Carolina, 27599, USA
}

\begin{abstract}
It is now widely recognized that dynamics are important to consider for understanding allosteric protein function. However, dynamics occur over a wide range of timescales, and how these different motions relate to one another is not well understood. Here, we report an NMR relaxation study of dynamics over multiple timescales at both backbone and side-chain sites upon an allosteric response to phosphorylation. The response regulator, Escherichia coli $\mathrm{CheY}$, allosterically responds to phosphorylation with a change in dynamics on both the $\mu \mathrm{s}-\mathrm{ms}$ timescale and ps-ns timescale. We observe an apparent decrease and redistribution of $\mu \mathrm{s}$-ms dynamics upon phosphorylation (and accompanying $\mathrm{Mg}^{2+}$ saturation) of CheY. Additionally, methyl groups with the largest changes in ps-ns dynamics localize to the regions of conformational change measured by $\mu$ s-ms dynamics. The limited spread of changes in ps-ns dynamics suggests a distinct relationship between motions on the $\mu \mathrm{s}-\mathrm{ms}$ and ps-ns timescales in CheY. The allosteric mechanism utilized by CheY highlights the diversity of roles dynamics play in protein function.
\end{abstract}

\section{Introduction}

Allostery is widespread in biology as an effective means for regulating protein activity. While the existence of protein dynamics over a range of timescales is well established, how these dynamics contribute to allosteric protein function is poorly understood. The mechanism(s) underlying long-range communication necessary for allostery may include not only large changes in the conformation but also changes in the dynamic fluctuations about a mean conformation. ${ }^{1,2}$ Allosteric mechanisms have been shown to utilize the now familiar conformational change, ${ }^{3-5}$ but more recent cases have been shown to solely use fast (i.e. psns) internal dynamics. ${ }^{6-8}$ It has been suggested that fast fluctuations are involved in facilitating the slower motions. ${ }^{9}$ This connection between different timescales of motion and relation to function may be an important mechanism in allostery. However, to our knowledge, only for adenylate kinase has there been any discussion of the relationship between timescales. ${ }^{9}$ Allosteric proteins often possess both slow and fast dynamics, but the relationship between the two remains unclear.

\footnotetext{
(C) 2013 Elsevier Ltd. All rights reserved.

Publisher's Disclaimer: This is a PDF file of an unedited manuscript that has been accepted for publication. As a service to our customers we are providing this early version of the manuscript. The manuscript will undergo copyediting, typesetting, and review of the resulting proof before it is published in its final citable form. Please note that during the production process errors may be discovered which could affect the content, and all legal disclaimers that apply to the journal pertain.
} 
Escherichia coli CheY is an ideal protein for the study of protein motions as they relate to function since it is an allosteric signaling protein that displays significant motions on multiple timescales. CheY is a response regulator (RR) from the two-component system that regulates chemotaxis. ${ }^{10,11}$ Upon phosphorylation at D57 ${ }^{12}$, CheY undergoes a conformational change which enables tight binding to the flagellar motor protein FliM (and minor interactions with $\mathrm{FliN}^{13}$ ) at a distal surface. CheY binding to FliM and FliN promotes a conformational change in the flagellar protein FliG which results in a switch from counterclockwise to clockwise flagellar rotation. ${ }^{14,15}$ Because of the necessity for a quick chemotactic response, the autodephosphorylation rate of CheY is $2.5 \mathrm{~min}^{-1} .{ }^{16}$ Therefore, the ability to study the phosphorylated state of CheY is difficult and it has become common to use the phosphoryl mimic $\mathrm{BeF}_{\mathrm{x}} \cdot{ }^{17-20}$

The CheY conformational switch has been extensively studied as a model RR for understanding phosphorylation induced activation. Phosphorylation in CheY is initially sensed by T87, A88 and K109 which form hydrogen bonds with the phosphoryl group and are important in the initial allosteric signaling. ${ }^{20,21}$ The phosphorylation-induced conformational change in CheY includes motion of the $\beta 4-\mathrm{a} 4$ loop and a quartet of residues (E89, W58, M85, Y106) ${ }^{22}$ whose motions result in the rotation of Y106 from an "out" solvent exposed rotamer to an "in" buried one (Fig. 1). ${ }^{18,20}$

Not only is CheY an excellent model RR for conformational change due to phosphorylation, RRs have become favored models for understanding conformational allostery. Previous studies of RRs focused on the conformational switch between inactive and active conformations on the slow timescale by monitoring the inherent equilibrium in the unphosphorylated protein. ${ }^{3,22-24}$ While fast backbone dynamics have also been reported, they have not been shown to be involved in the allosteric transition. ${ }^{3,24,25}$ Here, we report the first multi-timescale study of both an unphosphorylated and phosphorylated RR including measurement of fast side-chain dynamics.

We previously reported motions on the $\mu$ s-ms timescale that correlate with the intrinsic switching between inactive and active-like conformations in CheY in the absence of the activating phosphoryl group. ${ }^{22}$ Here we observe the slow dynamics dampen and shift to the FliM binding interface upon phosphorylation (and accompanying $\mathrm{Mg}^{2+}$ saturation) of CheY. Additionally, we show large changes in methyl dynamics on the ps-ns timescale upon phosphorylation of $\mathrm{CheY}$ correlate with areas that undergo conformational change. Additional small significant changes are located in other regions known to affect CheY function. The dynamics on the slow and fast timescales localize to the same areas indicating a possible connection between timescales that may be necessary for the allosteric transition in $\mathrm{CheY}$.

\section{Results and discussion}

\section{$\mu s-m s$ dynamics are dampened and shift toward the FliM binding interface upon phosphorylation of CheY}

${ }^{15} \mathrm{~N}$ Carr-Purcell-Meiboom-Gill (CPMG) relaxation dispersion experiments ${ }^{26}$ were used to measure motions on the $\mu \mathrm{s}-\mathrm{ms}$ timescale for unphosphorylated CheY (CheY-unP) ${ }^{22}$ and $\mathrm{BeF}_{\mathrm{x}}$-bound $\mathrm{CheY}$ (CheY-P). This experiment measures the contribution from a conformational exchange process on the $\mu$ s-ms timescale $\left(R_{\mathcal{E X}_{X}}\right)$ to the effective transverse relaxation rate $\left(R_{2, e f f}\right)$ as a function of the spacing between $180^{\circ}$ pulses in the CPMG train $\left(\tau_{\mathrm{cp}}\right)$

$$
R_{2, e f f}=R_{2}^{\circ}+R_{e x}\left({ }^{1} \tau_{c p}\right)
$$


where $R_{2}{ }^{\circ}$ is the intrinsic relaxation rate. ${ }^{27}$ Full relaxation dispersion experiments can be fit to reveal the kinetic and thermodynamic parameters of the exchange. For CheY-P, full relaxation dispersion experiments were carried out, but only a few residues have a high enough $R_{e X}\left(>2 \mathrm{~s}^{-1}\right)$ to enable accurate fitting and it is therefore more beneficial to only consider the level of $R_{e X}$ in each CheY state (details in Materials and Methods). The complete curves and fits for these residues $\left(R_{e X}>2 \mathrm{~s}^{-1}\right)$ can be found in the supplemental material (Fig. S1a).

Here, we compare $\mu$ s-ms dynamics in CheY-P to CheY-unP. CheY sample buffers with 10 $\mathrm{mM} \mathrm{Mg}{ }^{2+}$ were used, even though CheY-unP is not fully saturated with $\mathrm{Mg}^{2+}$ at this concentration $^{22}$ (CheY-P is), as partial saturation is expected under normal cellular conditions. Overall, there is a decrease in $R_{e X}$ upon phosphorylation of CheY (Fig. 2a) compared to CheY-unP in the presence of $10 \mathrm{mM} \mathrm{Mg}^{2+}$. Surprisingly, $R_{e X}$ is not zero for CheY-P. In CheY-unP, dynamics on this timescale report on the switch between inactive and active states (Fig. 2b). ${ }^{22}$ We would expect phosphorylated CheY to be fully switched to the active state and therefore have zero $R_{e X}$. However, we see small amounts of $R_{e X}$ throughout the protein and much of it localized to the $\beta 4-a 4$ loop and near to Y106 (Fig.2c). This indicates that CheY-P may be primarily in the active conformation but is able to switch to another conformation(s), such as the inactive state.

In CheY-unP, residues with dynamics on this timescale localize to the active site and allosteric pathways (Fig. 2b). Upon phosphorylation, residues with dynamics are shifted towards the FliM binding interface (Fig.2c). While the allosteric pathway continues to have a small amount of $R_{e X}$, many active site residues are diminished (Fig. S1b) and a new low level of $R_{e x}$ appears near to the FliM binding interface. The slow dynamics in CheY-P are indicative of its ability to access more than one conformation on the $\mu \mathrm{s}-\mathrm{ms}$ timescale. Since CheY does not switch to the active state in a two-state concerted manner ${ }^{22}$, it is not surprising that a different selection of residues show motions in the phosphorylated state. CheY-P is likely locally accessing the inactive conformation in the regions of the allosteric pathway. By contrast, the new dynamics of CheY-P at the FliM-binding interface are monitoring motions not present in CheY-unP and may be indicative of CheY-P's dynamics between multiple active-like states. It is possible that these are not new motions, but the timescale of the motions has shifted. If this were the case, motions in CheY-unP may have been too fast to monitor by these experiments, but upon phosphorylation the motions become slow enough to be detectable.

\section{Ps-ns dynamics become both more rigid and more flexible upon phosphorylation of CheY}

NMR ${ }^{15} \mathrm{~N}$ and ${ }^{2} \mathrm{H}$ relaxation experiments were collected for CheY-P and CheY-unP to measure dynamic fluctuations on the ps-ns timescale. The dynamics report on the N-H bond vector for ${ }^{15} \mathrm{~N}$ relaxation and the methyl symmetry axis of a $\mathrm{CH}_{2} \mathrm{D}$ methyl isotopomer for ${ }^{2} \mathrm{H}$ relaxation. Model free analysis of the relaxation parameters yields the order parameter $\left(S^{2}\right)$ for the bond vector which ranges from 0 (no orientational bias) to 1 (completely rigid). ${ }^{28,29}$ In addition to the order parameter, the effective correlation time $\left(\tau_{\mathrm{e}}\right)$ for the internal motion of the bond vector is obtained.

The backbone dynamics were measured utilizing ${ }^{15} \mathrm{~N} T_{1}, T_{2}$, and $\left\{{ }^{1} \mathrm{H}\right\}-{ }^{15} \mathrm{~N}$ NOE relaxation experiments. We found the CheY backbone to be rigid on the ps-ns timescale in areas of secondary structure. The order parameters for CheY-unP ranged from 0.66 to 0.94 with an average of 0.88 (Fig. S2) which is consistent with previously reported values. ${ }^{30}$ Upon phosphorylation, backbone dynamics only change slightly throughout the protein (Fig. S3). The small changes occur in both directions: some residues become more rigid and others become more flexible upon phosphorylation. 
It is becoming increasingly clear that side-chain dynamics can reveal functional dynamics that are invisible to the backbone since side-chain dynamics are more heterogeneous and more sensitive to perturbation..$^{31}{ }^{2} \mathrm{H}$ relaxation experiments ${ }^{32}$ were collected to measure the ps-ns dynamics of the methyl side chains of CheY-unP and CheY-P. The methyl order parameters $\left(S^{2}\right.$ axis $)$ ranged from 0.25 to 0.99 with an average of 0.71 for CheY-unP (Fig. S4). Comparison of CheY-unP and CheY-P order parameters show larger changes in order parameter than the backbone, with some methyl groups with increased flexibility and some with increased rigidity upon phosphorylation (Fig. 3a). Overall, it appears phosphorylation induces an slight increase in flexibility in CheY.

$\mathrm{T} 87$ and $\mathrm{A} 88$, which are located very close to the bound $\mathrm{BeF}_{\mathrm{x}}$, have increased rigidity upon phosphorylation. This is to be expected since binding pockets typically become more rigid upon ligand binding. Furthermore, the hydroxyl group of T87 and backbone of A88 form hydrogen bonds with the phosphate group. Therefore, it is not surprising that these particular methyl groups become more rigid. Other than at the active site, most changes in dynamics correspond to increases in flexibility upon phosphorylation. This is commonly seen and thought to compensate for the entropic penalty incurred by the rigidification of the active site residues. ${ }^{33,34}$ Many other residues exhibit changes in dynamics, although it is clear that much of the protein is silent (Fig. 3c).

Furthermore, some methyl groups have a change in the correlation time as measured by $\tau_{\mathrm{e}}$ (Fig. 3b) and many are not accompanied by a change in $S^{2}$ axis. Methyl groups with a change in $\tau_{\mathrm{e}}$ are distributed throughout the protein with many near to the active site and FliM binding interface (Fig. 3d). The methyl groups with the largest change in $\tau_{\mathrm{e}}, \mathrm{L} 28, \mathrm{~L} 43$, and A113 do not undergo changes in $S^{2}$ axis and are located far from the active site. This phenomenon was also observed upon mutation in eglin c. ${ }^{35} \mathrm{~A}$ large change in $\tau_{\mathrm{e}}$ without a change in $S_{\text {axis }}^{2}$ indicates a large change in the rate of rotation of the methyl group about the symmetry axis. Further interpretation of changes in $\tau_{\mathrm{e}}$ is unclear, yet it remains a reliable measure of the changes in dynamics on this timescale.

\section{The largest changes in ps-ns side-chain dynamics are along the allosteric pathway}

The allosteric switch in CheY and other RRs has been well characterized as a conformational change between inactive and active states. ${ }^{3,18,20,22-24}$ It is this conformational switch (which is triggered by phosphorylation) that allows increased activity at a distal site. In $\mathrm{CheY}$, the location of the conformational change as seen in numerous crystal structures ${ }^{18,36,37}$ and confirmed by numerous mutational studies ${ }^{16,38,39}$ centers around the initial sensing by T87 and A88. These residues couple to the movement of the $\beta 4-a 4$ loop (especially E89), rotation of W58 about $\chi_{2}$, rotation of M85 about $\chi_{1}$ and, most importantly, inward rotamer selection of Y106. This conformational switch can be monitored by NMR relaxation experiments (above).

The largest changes in side-chain methyl ps-ns dynamics upon phosphorylation occur in this region (indicated by the oval in Fig. 4). Not only are there changes at T87 and V107 (adjacent to Y106 motion), but also large changes at M85 and A88. In addition to V107, there are significant changes to 195 which contacts Y106, is involved in FliM binding, and alters CheY's activity when mutated. ${ }^{40}$ This further confirms the involvement of T87, A88, M85, Y106, and I95 in the conformational change and allosteric signaling upon phosphorylation.

The remainder of the $\beta 4$-a 4 loop shows a mixture of rigidification and flexibility upon activation. Although the ${ }^{2} \mathrm{H}$ methyl relaxation experiments yield no information on sidechain dynamics of E89, a small but detectable increase in the backbone $\mathrm{NH} S^{2}$ from $0.82 \pm$ 0.02 to $0.87 \pm 0.01$ was observed for this residue (Fig. S3), consistent with the formation of 
a hydrogen bond between the side-chain hydroxyl of Y106 and the backbone carbonyl of E89 upon activation ${ }^{18}$. Interestingly, the $\mathrm{C}^{\beta} S_{\text {axis }}^{2}$ of A90 is unusually low for both inactive and activated states, at a value of $\sim 0.67 \pm 0.01$, compared to the CheY average of 0.86 for alanine. No backbone $S^{2}$ values were obtained for A90 due to spectral overlap, but the low $S^{2}$ axis, as well as the low NH $S^{2}$ of $0.66 \pm 0.01$ for K91 (of the inactive state), suggest that A90 and K91 are quite flexible in the inactive state. Together, these data indicate that in general the $\beta 4-a 4$ loop is flexible, but rigidification of the backbone at E89 upon phosphorylation does not affect the flexibility of the $\mathrm{A} 90 \mathrm{C}^{\beta}$.

\section{Small significant changes in ps-ns side-chain dynamics are in distal regions associated with CheY function}

Many methyl groups with a small, but significant change in methyl order parameter or a change in correlation time are near to the active site yet are not known to be involved in the allosteric switch or coordinating the $\mathrm{Mg}^{2+}$ or $\mathrm{BeF}_{\mathrm{x}}$. For example, M17 and M60 have a small increase in $S^{2}$ axis and a small change in $\tau_{e}$, respectively. These residues are both near to the active site (Fig. 4b) but are not considered active site residues. In the CheY ${ }^{15} \mathrm{~N}-{ }^{1} \mathrm{H}$ HSQC, M60 is a broad peak and has a large chemical shift change upon $\mathrm{Mg}^{2+}$ binding and subsequent $\mathrm{BeF}_{\mathrm{x}}$ binding. Therefore, $\mathrm{M} 60$ reports on the binding of these two ligands. Since M17 has been shown to be intolerant of mutation, it was proposed to play a role in the propagation of signal after phosphorylation. ${ }^{38}$ Furthermore, mutation of methionine to alanine at 17 produces significant changes in the chemical shift of Y106 and V107 more than $9 \AA$ away (McDonald \& Lee, unpublished), indicating its involvement in the allosteric pathway. Both M17 and M60 are not known to be directly involved in the allosteric signaling upon phosphorylation, but both seem to have functional importance. Their significant changes in side-chain dynamics on the fast timescale may be indicative of their involvement in the allosteric signaling.

Another methyl group that has only a change in $\tau_{\mathrm{e}}$ without a change in $S^{2}$ axis is A113. A mutation of A113 to proline causes increased activity in CheY as measured by increased clockwise flagellar rotation and FliM binding affinity. ${ }^{39}$ Alternatively, a mutation to asparagine at this position results in an increase of counterclockwise flagellar rotation. A113 is more than $17 \AA$ from the bound $\mathrm{BeF}_{\mathrm{x}}$ and $12 \AA$ from the bound FliM peptide (using pdb $1 \mathrm{~F} 4 \mathrm{~V}^{19}$ ). Mutations that affect $\mathrm{CheY}$ function at A113 are surprising since it is so far from the active site, FliM, and any residues involved in the allosteric transition. Therefore, it is especially interesting to observe a change in ps-ns dynamics upon phosphorylation in the methyl group of A113. Other nearby methyl groups also have perturbations to dynamics, such as I20, L24, I55, and L120 (all decrease in $S_{\text {axis }}^{2}$, Fig. 3). All of these methyl groups localize to the pocket between the beta sheet and helices 1 and 5 . The residues form a network between the active site and the FliM binding interface (Fig. 4a). This suggests that the link between A113 and CheY function may be directly related to ps-ns dynamics associated with the side chains. To our knowledge, other than mutational studies of A113, this region of CheY or any RR has never been shown to be important for function.

\section{Correlation of fast and slow motions in CheY}

The relationship between the overall increase or decrease in flexibility of a protein on different timescales is not well understood. Here, by monitoring changes in both fast and slow dynamics upon phosphorylation, we hoped to shed light on this potentially complex relationship. Upon phosphorylation of $\mathrm{CheY}$, we report an apparent decrease in slow dynamics with an overall slight increase in flexibility in the side chains on the fast timescale. However, we do observe an apparent increase in rigidity on both timescales in the active site. This complex behavior in CheY demonstrates the difficulty in predicting the relationship between motions on different timescales. 
Recent reports of changes in side-chain dynamics on the fast timescale link these changes to the function of the protein. These reports rely on the connection between fast dynamics and conformational entropy. ${ }^{6,41-43}$ For calmodulin, ${ }^{6}$ a PDZ domain, ${ }^{7}$ and catabolite activator protein ${ }^{8}$ changes in conformational entropy regulate the protein's binding activity. In these cases, changes in the dynamics were detected throughout the protein as opposed to being localized to the active site. This global redistribution of side-chain motions has an overall effect on the conformational entropy of the system to affect protein function. Here, we report the changes in ps-ns dynamics of CheY are largely localized to the active site and residues in the allosteric pathway. In CheY, at least for the case of phosphorylation, there is not a global effect on the motions of the methyl side chains on the fast timescale as seen in these other cases.

In addition to the spatial restriction of changes in fast side-chain dynamics in CheY, there is segregation of residues that become more rigid from those that become more flexible. The largest rigidifications to ps-ns side-chain dynamics are in the active site with residues that become more flexible distributed nearby (Fig. 3c). This localization of rigidifying residues may be necessary for the allosteric conformational switch in CheY. 9 of 13 residues with a change in ps-ns dynamics become more flexible upon phosphorylation and this is likely to entropically compensate for the large rigidifications in the active site. ${ }^{33,}{ }^{34}$ Furthermore, CheY seems to be built for this kind of entropic compensation. All four methyl groups that become more rigid upon phosphorylation have low $S_{\text {axis }}^{2}$ values in CheY-unP compared to the average values for that particular methyl group. Most interesting is $\mathrm{T} 87 \mathrm{C}^{2}$ which has the largest increase in $S_{\text {axis }}^{2}$ and has an $S^{2}$ axis value of $0.50 \pm 0.04$ in CheY-unP where the average CheY values for Thr $\mathrm{C}^{2}$ is 0.69 . In the unphosphorylated state of CheY, many methyl groups, especially $\mathrm{T} 87 \mathrm{C}^{2}$ seem "poised" for the change in ps-ns dynamics upon phosphorylation. Residues that become more flexible do not seem to be "poised" ( 3 of 9 have high $S_{\text {axis }}^{2}$ values in CheY-unP), but nearly all (8 of 9) have less than average $S_{\text {axis }}^{2}$ values in CheY-P. Together, this suggests the entropic compensation in CheY may be a necessary element of the allosteric switch.

While the entropic compensation may be necessary in $\mathrm{CheY}$, changes in the ps-ns dynamics caused by phosphorylation seem to be restricted to locations near to the active site. Many distal regions of CheY are silent on the $\mu$ s-ms timescale and have little or no change in fast dynamics upon phosphorylation. For proteins that have been similarly studied on multiple timescales ${ }^{8,44}$, there is a global impact on ps-ns dynamics upon perturbation. These proteins (in addition to others with reports of changes in ps-ns timescale dynamics upon perturbation ${ }^{6,7}$ ) seem to readily spread changes in ps-ns dynamics throughout the protein. CheY's unique ability to subdue this propagation of ps-ns dynamics changes may reflect a distinct mechanism by which proteins utilize ps-ns dynamics for function.

Localization of changes in fast dynamics at regions of allosteric conformational change is not surprising. This is due to the conformational change which causes a change in the chemical environment of a methyl group and the very responsive ps-ns side-chain dynamics being affected. In CheY, residues A113, M60, and M17 are in regions without large conformational change and do not have measureable slow motions. Nevertheless, the presence of modest changes in ps-ns side-chain dynamics at these residues suggests changes in ps-ns dynamics in CheY go beyond the simple explanation of a mere effect from conformational change.

CheY reveals a mechanism that utilizes segmental motions on the timescale of the conformational change $\mathrm{e}^{22}$ and colocalized changes in faster fluctuations upon that conformational change. This correlation of motions on different timescales in CheY sheds light on the importance of the relationship between multiple timescales of motion. The 
nature of the relationship and how it results in allosteric function is still poorly understood. Utilization of CheY mutants may yield additional information to better understand the relationship between timescales in an allosteric signaling protein.

\section{Materials and Methods}

\section{Protein expression and purification}

E. coli CheY was overexpressed and purified as described previously. ${ }^{22}$ The CheY plasmid was transformed into BL21 Star (DE3) cells (Invitrogen) and grown in minimal media supplemented with the appropriate isotopes for the desired labeling utilizing ${ }^{15} \mathrm{NH}_{4} \mathrm{Cl}$ [99\%] and/or D-glucose [U-13 $\mathrm{C}_{6}-99 \%$ ]. Samples for relaxation dispersion and ${ }^{2} \mathrm{H}$ relaxation were grown to yield $>80 \%$ and $\sim 60 \%{ }^{2} \mathrm{H}$ incorporation, respectively. Pure CheY was concentrated and stored in NMR buffer ( $\left.50 \mathrm{mM} \mathrm{NaP}_{\mathrm{i}}, 10 \mathrm{mM} \mathrm{MgCl}_{2}, 0.02 \% \mathrm{NaN}_{3}, \mathrm{pH} 7.0\right)$ at $4{ }^{\circ} \mathrm{C}$.

\section{Preparation of $\mathrm{BeF}_{\mathrm{x}}$-bound CheY}

As previously described ${ }^{22}, \mathrm{BeF}_{\mathrm{x}}$-bound $\mathrm{CheY}$ was prepared with $4 \mathrm{mM} \mathrm{BeCl}_{2}$ and $25 \mathrm{mM}$ $\mathrm{NaF}$ to ensure saturation of $1 \mathrm{mM} \mathrm{CheY}$ and in an ideal ratio for a $\mathrm{BeF}_{3}$ complex. Assuming a $K_{\mathrm{d}}$ of $7.7 \mu \mathrm{M}$ for $\mathrm{BeF}_{\mathrm{x}}$ and $\mathrm{CheY}^{45}, 99.7 \%$ of $\mathrm{CheY}$ is bound by $\mathrm{BeF}_{\mathrm{X}}$. The NMR samples were prepared by starting with a typical CheY-unP sample $\left(1 \mathrm{mM} \mathrm{CheY}\right.$ and $10 \%{ }^{2} \mathrm{H}_{2} \mathrm{O}$ in NMR buffer) and adding the $\mathrm{BeCl}_{2}$ and lastly $\mathrm{NaF}$. Samples were left at room temperature overnight and centrifuged briefly before transferring to an NMR tube.

\section{NMR Spectroscopy}

All NMR spectra were collected on $1 \mathrm{mM} \mathrm{CheY} \mathrm{in} \mathrm{NMR} \mathrm{buffer} \mathrm{and} 10 \%{ }^{2} \mathrm{H}_{2} \mathrm{O}$ at $25{ }^{\circ} \mathrm{C}$ except for relaxation dispersion experiments which were collected at $15{ }^{\circ} \mathrm{C}$. NMR experiments were collected on Varian INOVA spectrometers equipped with roomtemperature $(500$ and $600 \mathrm{MHz})$ or cryogenic $(700 \mathrm{MHz})$ probes. Side-chain methyl assignments were made by utilizing previously assigned $\mathrm{C}^{a}$ and $\mathrm{C}^{\beta}$ chemical shifts and a three-dimensional $\mathrm{HCCH}_{3}$ total correlated spectroscopy spectrum. ${ }^{46}$ Resonances from methionine methyl groups were assigned using a combination of a three-dimensional methyl-methyl NOESY spectrum ${ }^{47}$ and mutation of methionine residues. All NMR data were processed using NMRPipe ${ }^{48}$ and visualized with NMRDraw and NMRView ${ }^{49}$.

\section{${ }^{15} \mathrm{~N}$ CPMG relaxation dispersion}

${ }^{15} \mathrm{~N}$ relaxation dispersion of CheY-P was performed using a relaxation-compensated CPMG experiment ${ }^{27}$ utilizing a $20 \mathrm{~ms}$ total CPMG period. Ten $\tau_{\mathrm{cp}}$ values with two duplicates and a reference experiment were collected interleaved at $700 \mathrm{MHz} . R_{2, \text { eff }}$ was calculated from peak intensities as described previously ${ }^{50}$. Approximate $R_{e_{X}}$ was calculated by using $R_{2, \text { eff }}$ values from this experiment: Approximate $R_{e X}=R_{2, \text { eff }}\left(\tau_{\mathrm{cp}}=5 \mathrm{~ms}\right)-R_{2, \text { eff }}\left(\tau_{\mathrm{cp}}=0.556 \mathrm{~ms}\right)$. Error in approximate $R_{e X}$ was propagated from peak intensity analysis of duplicate $\tau_{\mathrm{cp}}$ values. Relaxation dispersion of CheY-unP was previously published ${ }^{22}$ and approximate $R_{e X}$ values were calculated in the same manner as CheY-P for data collected at $700 \mathrm{MHz}$.

\section{${ }^{15} \mathrm{~N}$ backbone and ${ }^{2} \mathrm{H}$ methyl relaxation}

Standard backbone ${ }^{15} \mathrm{~N}$ relaxation experiments were used to collect the $T_{1}, T_{2}$, and $\left\{{ }^{1} \mathrm{H}\right\}-{ }^{15} \mathrm{~N}$ NOE data at $500 \mathrm{MHz}$ for CheY-unP and at 500 and $600 \mathrm{MHz}$ for CheY-P ${ }^{51}$. For $T_{1}$ and $T_{2}$ experiments, 9 or 10 relaxation time points along with 3 duplicates were collected. For the $\left\{{ }^{1} \mathrm{H}\right\}-{ }^{15} \mathrm{~N}$ NOE, the recycle delay was a combination of a ${ }^{1} \mathrm{H}$ saturation time of 5.5 or $6 \mathrm{~s}$ and a pre-delay of $100 \mathrm{~ms} .{ }^{2} \mathrm{H} T_{1}$ and $T_{1} \rho$ experiments ${ }^{32}$ to measure $I_{\mathrm{z}} C_{\mathrm{z}} D_{\mathrm{z}}$ and $I_{\mathrm{z}} C_{\mathrm{z}} D_{\mathrm{z}}$ relaxation ${ }^{32}$ for side-chain ps-ns dynamics were collected at 500 and 700 
$\mathrm{MHz}$ for CheY-unP and at 500 and $600 \mathrm{MHz}$ for CheY-P. For each experiment, 9 relaxation time points along with 3 duplicate points were collected.

\section{Relaxation analysis}

Relaxation decays were best fit to single exponentials using in-house programs. Motions on the ps-ns timescale were characterized using the model-free formalism. ${ }^{28,}{ }^{29} \mathrm{Global}$ correlation times for both CheY-unP and CheY-P were determined to be $8.1 \mathrm{~ns}$ from global analysis of the data. Backbone relaxation rates were fitted to the five standard model-free models and the Akaike information criterion ${ }^{53}$ was used for model selection utilizing the inhouse program relxn2.2. ${ }^{35}$ Determination of side-chain methyl dynamics parameters (order parameter, $S_{\text {axis }}^{2}$ and effective correlation time, $\tau_{\mathrm{e}}$ ) was carried out as previously described. ${ }^{54}$

\section{Supplementary Material}

Refer to Web version on PubMed Central for supplementary material.

\section{Acknowledgments}

This work was supported by National Institutes of Health (NIH) grant GM066009 (to A.L.L.), and additional support was provided to L.R.M. through NIH training grant GM008570.

\section{References}

1. Cui Q, Karplus M. Allostery and cooperativity revisited. Protein Sci. 2008; 17:1295-307. [PubMed: 18560010]

2. Manley G, Loria JP. NMR insights into protein allostery. Arch. Biochem. Biophys. 2012; 519:22331. [PubMed: 22198279]

3. Volkman BF, Lipson D, Wemmer DE, Kern D. Two-state allosteric behavior in a single-domain signaling protein. Science. 2001; 291:2429-2433. [PubMed: 11264542]

4. Farber PJ, Mittermaier A. Concerted dynamics link allosteric sites in the PBX homeodomain. J. Mol. Biol. 2011; 405:819-30. [PubMed: 21087615]

5. Bruschweiler S, Schanda P, Kloiber K, Brutscher B, Kontaxis G, Konrat R, Tollinger M. Direct observation of the dynamic process underlying allosteric signal transmission. J. Am. Chem. Soc. 2009; 131:3063-8. [PubMed: 19203263]

6. Frederick KK, Marlow MS, Valentine KG, Wand AJ. Conformational entropy in molecular recognition by proteins. Nature. 2007; 448:325-9. [PubMed: 17637663]

7. Petit CM, Zhang J, Sapienza PJ, Fuentes EJ, Lee AL. Hidden dynamic allostery in a PDZ domain. Proc. Natl. Acad. Sci. USA. 2009; 106:18249-18254. [PubMed: 19828436]

8. Tzeng SR, Kalodimos CG. Protein activity regulation by conformational entropy. Nature. 2012; 488:236-240. [PubMed: 22801505]

9. Henzler-Wildman KA, Lei M, Thai V, Kerns SJ, Karplus M, Kern D. A hierarchy of timescales in protein dynamics is linked to enzyme catalysis. Nature. 2007; 450:913-U27. [PubMed: 18026087]

10. Clegg DO, Koshland DE Jr. The role of a signaling protein in bacterial sensing: behavioral effects of increased gene expression. Proc. Natl. Acad. Sci. USA. 1984; 81:5056-60. [PubMed: 6089173]

11. Matsumura P, Rydel JJ, Linzmeier R, acante D. Overexpression and sequence of the Escherichia Coli CheY gene and biochemical activities of the CheY protein. J. Bacteriol. 1984; 160:36-41. [PubMed: 6090423]

12. Sanders DA, Gillece-Castro BL, Stock AM, Burlingame AL, Koshland DE Jr. Identification of the site of phosphorylation of the chemotaxis response regulator protein, CheY. J. Biol. Chem. 1989; 264:21770-8. [PubMed: 2689446] 
13. Sarkar MK, Paul K, Blair D. Chemotaxis signaling protein CheY binds to the rotor protein FliN to control the direction of flagellar rotation in Escherichia coli. Proc. Natl. Acad. Sci. USA. 2010; 107:9370-5. [PubMed: 20439729]

14. Baker MD, Wolanin PM, Stock JB. Signal transduction in bacterial chemotaxis. Bioessays. 2006; 28:9-22. [PubMed: 16369945]

15. Paul K, Brunstetter D, Titen S, Blair DF. A molecular mechanism of direction switching in the flagellar motor of Escherichia coli. Proc. Natl. Acad. Sci. USA. 2011; 108:17171-6. [PubMed: 21969567]

16. Thomas SA, Brewster JA, Bourret RB. Two variable active site residues modulate response regulator phosphoryl group stability. Mol. Microbiol. 2008; 69:453-65. [PubMed: 18557815]

17. Yan D, Cho HS, Hastings CA, Igo MM, Lee SY, Pelton JG, Stewart V, Wemmer DE, Kustu S. Beryllofluoride mimics phosphorylation of $\mathrm{NtrC}$ and other bacterial response regulators. Proc. Natl. Acad. Sci. USA. 1999; 96:14789-14794. [PubMed: 10611291]

18. Lee SY, Cho HS, Pelton JG, Yan D, Berry EA, Wemmer DE. Crystal structure of activated CheY. Comparison with other activated receiver domains. J. Biol. Chem. 2001; 276:16425-31. [PubMed: 11279165]

19. Lee SY, Cho HS, Pelton JG, Yan D, Henderson RK, King DS, Huang L, Kustu S, Berry EA, Wemmer DE. Crystal structure of an activated response regulator bound to its target. Nat. Struct. Biol. 2001; 8:52, 6. [PubMed: 11135671]

20. Cho HS, Lee SY, Yan D, Pan X, Parkinson JS, Kustu S, Wemmer DE, Pelton JG. NMR structure of activated CheY. J. Mol. Biol. 2000; 297:543-51. [PubMed: 10731410]

21. Appleby JL, Bourret RB. Proposed signal transduction role for conserved CheY residue Thr87, a member of the response regulator active-site quintet. J. Bacteriol. 1998; 180:3563-9. [PubMed: 9657998]

22. McDonald LR, Boyer JA, Lee AL. Segmental motions, not a two-state concerted switch, underlie allostery in CheY. Structure. 2012; 20:1363-1373. [PubMed: 22727815]

23. Gardino AK, Villali J, Kivenson A, Lei M, Liu CF, Steindel P, Eisenmesser EZ, Labeikovsky W, Wolf-Watz M, Clarkson MW, Kern D. Transient non-native hydrogen bonds promote activation of a signaling protein. Cell. 2009; 139:1109-1118. [PubMed: 20005804]

24. Feher VA, Cavanagh J. Millisecond-timescale motions contribute to the function of the bacterial response regulator protein Spo0F. Nature. 1999; 400:289, 93. [PubMed: 10421374]

25. Sheftic SR, Garcia PP, White E, Robinson VL, Gage DJ, Alexandrescu AT. Nuclear magnetic resonance structure and dynamics of the response regulator Sma0114 from Sinorhizobium meliloti. Biochemistry. 2012; 51:6932-41. [PubMed: 22880754]

26. Palmer AG, Kroenke CD, Loria JP. Nuclear magnetic resonance methods for quantifying microsecond-to-millisecond motions in biological macromolecules. Methods Enzymol. 2001; 339:204-238. [PubMed: 11462813]

27. Loria JP, Rance M, Palmer AG. A relaxation-compensated Carr-Purcell-Meiboom-Gill sequence for characterizing chemical exchange by NMR spectroscopy. J. Am. Chem. Soc. 1999; 121:23312332.

28. Lipari G, Szabo A. Model-free approach to the interpretation of nuclear magnetic resonance relaxation in macromolecules.1. Theory and range of validity. J. Am. Chem. Soc. 1982; 104:45464559.

29. Lipari G, Szabo A. Model-free approach to the interpretation of nuclear magnetic resonance relaxation in macromolecules. 2. Analysis of experimental results. J. Am. Chem. Soc. 1982; 104:4559-4570.

30. Moy FJ, Lowry DF, Matsumura P, Dahlquist FW, Krywko JE, Domaille PJ. Assignments, secondary structure, global fold, and dynamics of chemotaxis-Y protein using 3-dimensional and 4-dimensional heteronuclear (C-13,N-15) NMR spectroscopy. Biochemistry. 1994; 33:1073110742. [PubMed: 8075074]

31. Igumenova TI, Frederick KK, Wand AJ. Characterization of the fast dynamics of protein amino acid side chains using NMR relaxation in solution. Chem. Rev. 2006; 106:1672-99. [PubMed: 16683749] 
32. Muhandiram DR, Yamazaki T, Sykes BD, Kay LE. Measurement of H-2 T-1 and T-1p relaxationtimes in uniformly C-13-labeled and fractionally H-2-labeled proteins in solution. J. Am. Chem. Soc. 1995; 117:11536-11544.

33. Lee AL, Kinnear SA, Wand AJ. Redistribution and loss of side chain entropy upon formation of a calmodulin-peptide complex. Nat. Struct. Biol. 2000; 7:72-77. [PubMed: 10625431]

34. Loh AP, Pawley N, Nicholson LK, Oswald RE. An increase in side chain entropy facilitates effector binding: NMR characterization of the side chain methyl group dynamics is $\mathrm{Cdc} 42 \mathrm{Hs}$. Biochemistry. 2001; 40:4590-4600. [PubMed: 11294626]

35. Clarkson MW, Gilmore SA, Edgell MH, Lee AL. Dynamic coupling and allosteric behavior in a nonallosteric protein. Biochemistry. 2006; 45:7693-9. [PubMed: 16784220]

36. Simonovic M, Volz K. A distinct meta-active conformation in the 1.1-A resolution structure of wild-type ApoCheY. J. Biol. Chem. 2001; 276:28637-40. [PubMed: 11410584]

37. Dyer CM, Dahlquist FW. Switched or not?: the structure of unphosphorylated CheY bound to the N terminus of FliM. J. Bacteriol. 2006; 188:7354-63. [PubMed: 17050923]

38. Smith JG, Latiolais JA, Guanga GP, Citineni S, Silversmith RE, Bourret RB. Investigation of the role of electrostatic charge in activation of the Escherichia coli response regulator CheY. J. Bacteriol. 2003; 185:6385-6391. [PubMed: 14563873]

39. Smith JG, Latiolais JA, Guanga GP, Pennington JD, Silversmith RE, Bourret RB. A search for amino acid substitutions that universally activate response regulators. Mol. Microbiol. 2004; 51:887-901. [PubMed: 14731287]

40. Schuster M, Zhao R, Bourret RB, Collins EJ. Correlated switch binding and signaling in bacterial chemotaxis. J. Biol. Chem. 2000; 275:19752-8. [PubMed: 10748173]

41. Akke M, Bruschweiler R, Palmer AG. Nmr Order Parameters and Free-Energy - an Analytical Approach and Its Application to Cooperative Ca2+ Binding by Calbindin-D(9k). J. Am. Chem. Soc. 1993; 115:9832-9833.

42. Yang D, Kay LE. Contributions to conformational entropy arising from bond vector fluctuations measured from NMR-derived order parameters: application to protein folding. J. Mol. Biol. 1996; 263:369-82. [PubMed: 8913313]

43. Li Z, Raychaudhuri S, Wand AJ. Insights into the local residual entropy of proteins provided by NMR relaxation. Protein Sci. 1996; 5:2647-50. [PubMed: 8976574]

44. Schnell JR, Dyson HJ, Wright PE. Effect of cofactor binding and loop conformation on side chain methyl dynamics in dihydrofolate reductase. Biochemistry. 2004; 43:374-83. [PubMed: 14717591]

45. Schuster M, Silversmith RE, Bourret RB. Conformational coupling in the chemotaxis response regulator CheY. Proc. Natl. Acad. Sci. USA. 2001; 98:6003-8. [PubMed: 11353835]

46. Uhrin D, Uhrinova S, Leadbeater C, Nairn J, Price NC, Barlow PN. 3D HCCH(3)-TOCSY for resonance assignment of methyl-containing side chains in (13)C-labeled proteins. J. Magn. Reson. 2000; 142:288-93. [PubMed: 10648145]

47. Zwahlen C, Gardner KH, Sarma SP, Horita DA, Byrd RA, Kay LE. An NMR experiment for measuring methyl-methyl NOEs in C-13-labeled proteins with high resolution. J. Am. Chem. Soc. 1998; 120:7617-7625.

48. Delaglio F, Grzesiek S, Vuister GW, Zhu G, Pfeifer J, Bax A. NMRPipe: a multidimensional spectral processing system based on UNIX pipes. J. Biomol. NMR. 1995; 6:277-93. [PubMed: 8520220]

49. Johnson BA. Using NMRView to visualize and analyze the NMR spectra of macromolecules. Methods Mol. Biol. 2004; 278:313-52. [PubMed: 15318002]

50. Mulder FA, Mittermaier A, Hon B, Dahlquist FW, Kay LE. Studying excited states of proteins by NMR spectroscopy. Nat. Struct. Biol. 2001; 8:932-5. [PubMed: 11685237]

51. Farrow NA, Muhandiram R, Singer AU, Pascal SM, Kay CM, Gish G, Shoelson SE, Pawson T, Formankay JD, Kay LE. Backbone Dynamics of a Free and a Phosphopeptide-Complexed Src Homology-2 Domain Studied by N-15 Nmr Relaxation. Biochemistry. 1994; 33:5984-6003. [PubMed: 7514039]

52. Millet O, Muhandiram DR, Skrynnikov NR, Kay LE. Deuterium spin probes of side-chain dynamics in proteins. 1. Measurement of five relaxation rates per deuteron in (13)C-labeled and 
fractionally (2)H-enriched proteins in solution. J. Am. Chem. Soc. 2002; 124:6439-48. [PubMed: 12033875]

53. d'Auvergne EJ, Gooley PR. The use of model selection in the model-free analysis of protein dynamics. J. Biomol. NMR. 2003; 25:25-39. [PubMed: 12566997]

54. Fuentes EJ, Der CJ, Lee AL. Ligand-dependent dynamics and intramolecular signaling in a PDZ domain. J. Mol. Biol. 2004; 335:1105-1115. [PubMed: 14698303] 


\section{Highlights}

- CheY switches between inactive and active conformations on the $\mu$ s-ms timescale.

- Phosphorylation dampens and redistributes $\mu \mathrm{s}-\mathrm{ms}$ motions in CheY.

- Phosphorylation induces changes in ps-ns side-chain dynamics in CheY.

- We observe limited global spreading of ps-ns dynamic changes in CheY.

- Ps-ns dynamic changes localize to the allosteric conformational change region. 

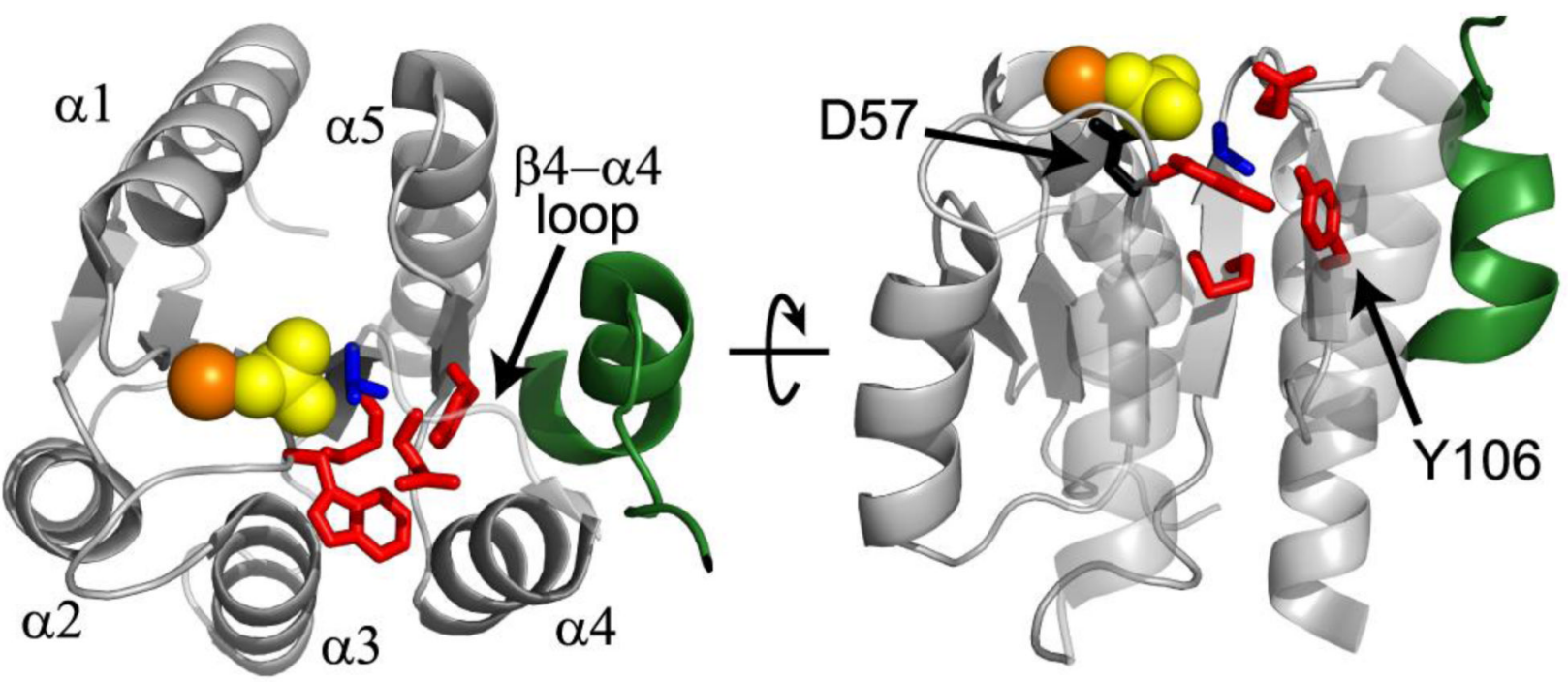

Fig. 1.

Crystal structure of CheY displaying residues involved in the conformational switch. FliM (green), $\mathrm{BeF}_{\mathrm{X}}$ (yellow), and $\mathrm{Mg}^{2+}$ (orange)-bound CheY $\left(1 \mathrm{~F} 4 \mathrm{~V}^{19}\right)$. The site of phosphorylation, D57, is highlighted in black. Allosteric signaling quartet, W58, M85, E89, and Y106, are shown as red sticks and T87 as blue sticks. 

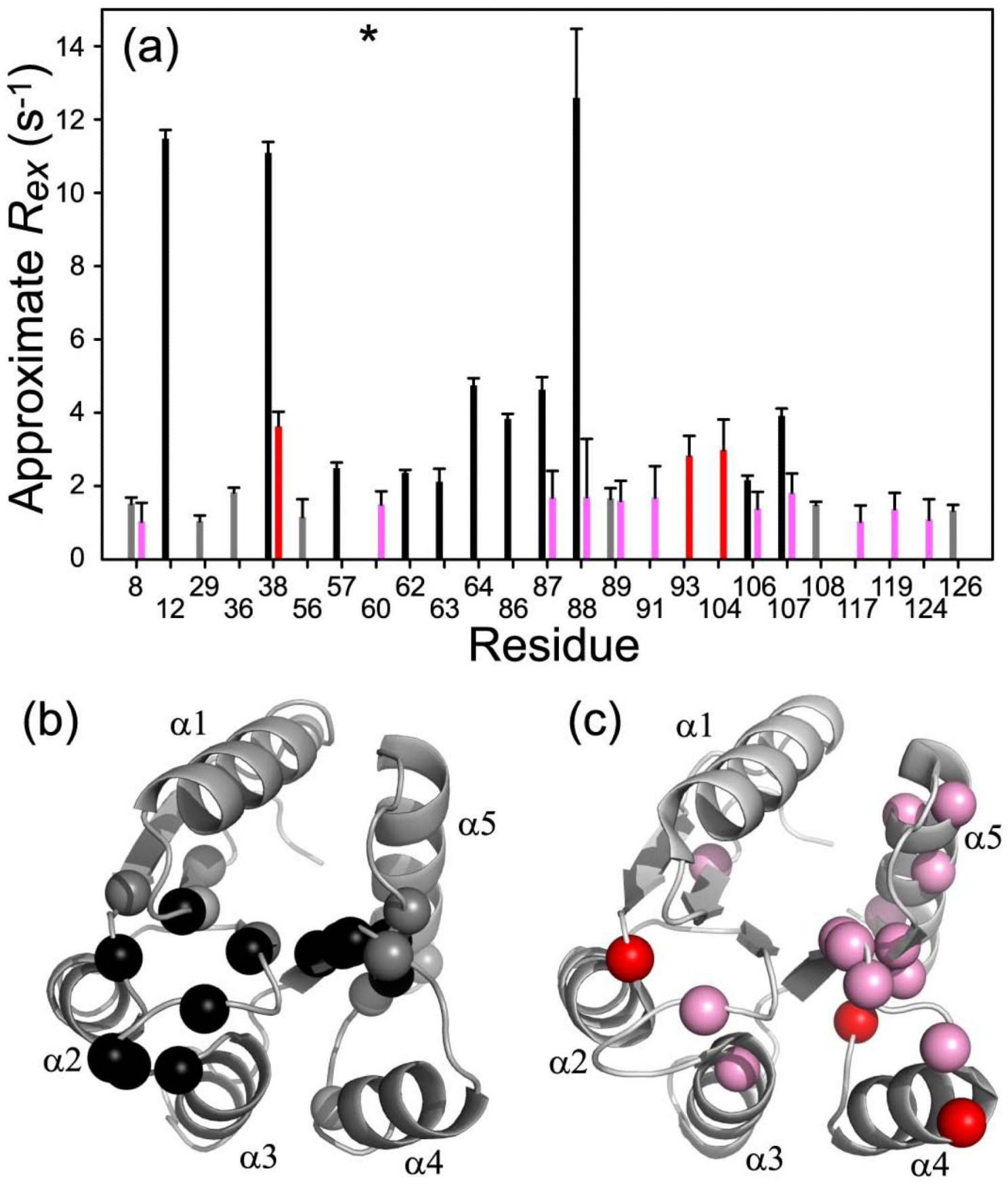

Fig. 2.

Comparison of $\mu$ s-ms motions between CheY-unP (black/grey) and CheY-P (red/pink). Approximate $R_{e X}$ is shown as bars (a) and displayed on the crystal structure of CheY-unP (b) and CheY-P (c). Black or red indicates $R_{e X}>2 \mathrm{~s}^{-1}$ and grey or pink indicates $2 \mathrm{~s}^{-1}>R_{e X}$ $>1 \mathrm{~s}^{-1}$. $R_{e X}$ below $1 \mathrm{~s}^{-1}$ is considered zero and not shown. Residues are displayed if $R_{e X}$ is greater than $1 \mathrm{~s}^{-1}$ for either CheY-unP or CheY-P. Approximate $R_{e X}=R_{2 e f f}\left(\tau_{\mathrm{cp}}=5 \mathrm{~ms}\right)-$ $R_{2 \text { eff }}\left(\tau_{\mathrm{cp}}=0.556 \mathrm{~ms}\right)$ at $700 \mathrm{MHz}$. For both CheY-unP and CheY-P, an F-test $\left(\mathrm{a}_{\text {critical }}=\right.$ 0.10 ) was used to remove any residues with unreliable $R_{e_{X}}$ values by fitting to both a straight line and a simple two-state model. M60 failed the F-test indicated by an asterisk for CheYunP. 



Fig. 3.

Difference in ps-ns motions between CheY-unP and CheY-P. Change in the order parameter (a) and correlation time (b) upon phosphorylation with colored bars shown as colored spheres in (c) and (d), respectively. $\Delta S_{\text {axis }}^{2}=S_{\text {axis }}^{2}\left(\right.$ CheY-P) $-S_{\text {axis }}^{2}$ (CheY-unP) and $\Delta \tau_{\mathrm{e}}=$ $\tau_{\mathrm{e}}(\mathrm{CheY}-\mathrm{P})-\tau_{\mathrm{e}}(\mathrm{CheY}$-unP). Blue indicates an increase in rigidity upon phosphorylation, and green is an increase in flexibility. Colored bars indicate a significant change determined by $\Delta S_{\text {axis }}^{2}>2 \sigma$ or $\tau_{\mathrm{e}}>2 \sigma$. 

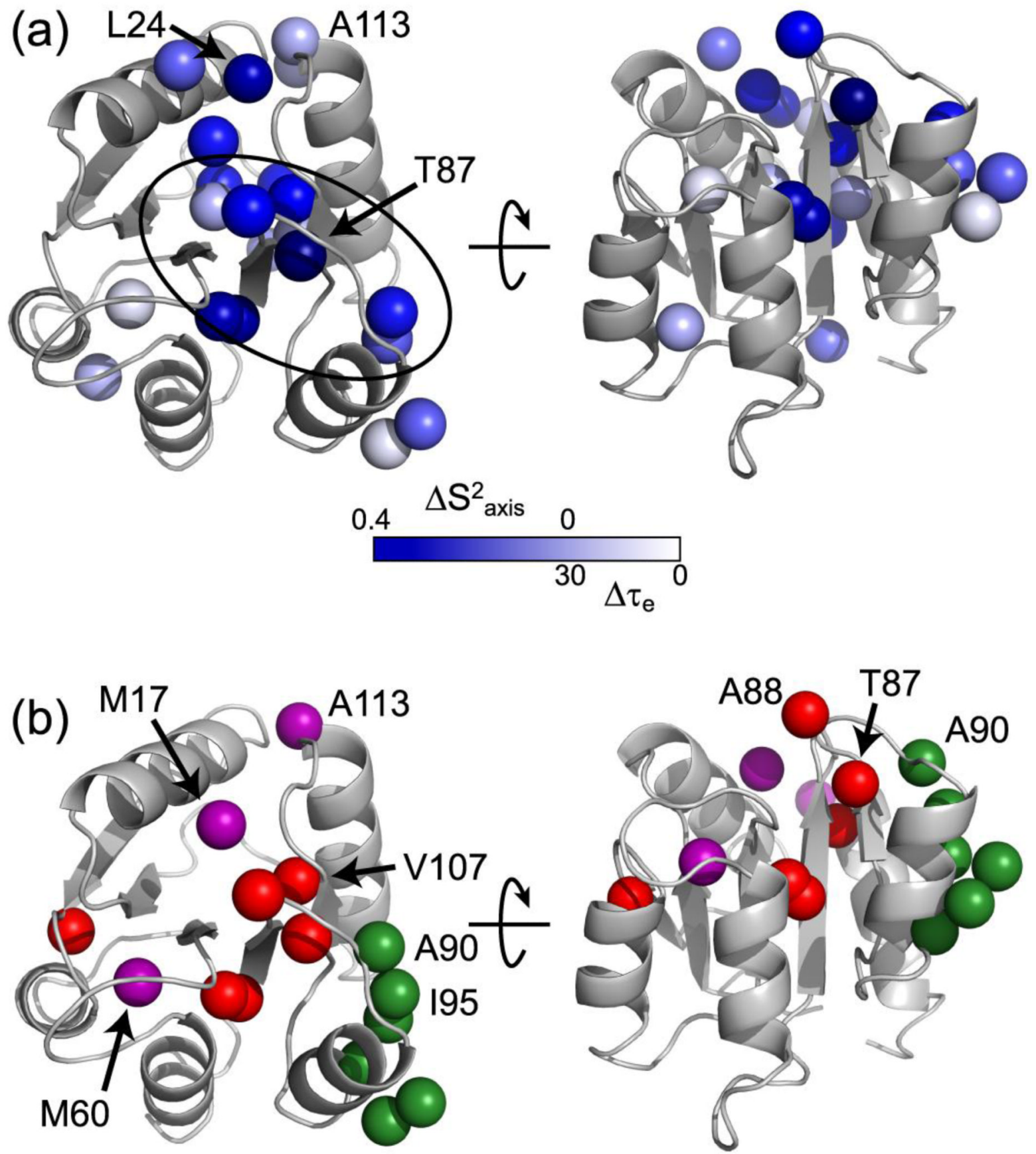

Fig. 4.

Largest changes in ps-ns dynamics localize to the region of allosteric conformational change. (a) Methyl groups with a change in ps-ns dynamics upon phosphorylation are shown as blue spheres. A gradient color scheme is used in which the largest $\Delta S^{2}$ axis are darkest blue and lightest colors indicate a change in $\tau_{\mathrm{e}}$ without a change in $\Delta S^{2}$ axis. Residues with a change in both $S^{2}$ axis and $\tau_{\mathrm{e}}$ are shown according to $\Delta S^{2}$ axis. A region that contains many large changes in $\Delta S^{2}$ axis and $\tau_{\mathrm{e}}$ that corresponds to the allosteric conformational switch are enclosed by the oval. (b) The locations of residues active in the allosteric switch (red), FliM binding (green), or mutation (purple) have their methyl groups shown as spheres. 